\title{
La ricerca in Psicologia Clinica Perinatale: fattori di rischio e protezione per la tutela della salute mentale
}

\author{
L. Cena, A. Imbasciati
}

\subsection{I multiformi aspetti della perinatalità psichica: che cosa esplorare in un'indagine per la tutela della salute mentale?}

Nell'ambito della ricerca in Psicologia Clinica Perinatale, in una prospettiva dinamico-clinica e diacronica si fa riferimento al concetto di "perinatalità psichica" (capitolo 2) non tanto per lo sviluppo del bimbo, quanto come perinatalità della psiche dei genitori: un "segmento" del processo di genitorialità presente in diverse forme evolutive in ogni età della vita di ogni individuo e nella sua più evidente manifestazione in prossimità di un progetto di filiazione (Missioner, 2005). Come evidenziato nel precedente capitolo la perinatalità psichica implica un periodo tra l'ipotetico progetto generativo/genitoriale (Stoleru, 1995), la sua pianificazione e la sua realizzazione, fino al primo sviluppo del bambino: questa perinatalità psichica costituisce una delle prime sequenze del processo di genitorialità. Che cosa esplorare allora in un'indagine per la salute mentale perinatale?

Donald Winnicott scriveva che "i bambini nascono quando vengono pensati" (Winnicott, 1987). Ma quando e come nascono i genitori? Prima che nascano i loro bambini, oppure quando "iniziano a pensare" ai loro bambini? Oppure quando questi sono in gestazione, o ancora quando, una volta nati, questi richiederanno loro accudimento, cure e protezione?

C'è un figlio segreto, autogenerato nell'immmaginario individuale della propria infanzia, il "figlio della notte" (Vegetti-Finzi, 1990) e c'è un figlio che prende origine nella mente della coppia. La nascita di un figlio ha inizio nel vissuto genitoriale a partire dal momento della decisione/programmazione che la coppia fa sul

L. Cena $(\square)$

Professore Associato di Psicologia Clinica

Dipartimento Materno Infantile, Facoltà di Medicina e Chirurgia

Università degli Studi di Brescia

cena@med.unibs.it 
proprio figlio futuro (Capodieci et al., 1990; De Renoche, Della Giustina, 1989). Ma, a quest'epoca, il genitore è già "nato"?

L'ingresso reale nella genitorialità è una svolta irreversibile: ma a quest'epoca, a livello psichico, c'è già un genitore nelle persone che mettono al mondo un figlio? La coppia viene di fatto investita di nuovi ruoli e compiti legati al "far posto" non solo fisicamente (nel ventre della madre), ma soprattutto emotivamente, nella propria mente, al nuovo venuto: la loro vita di sempre non sarà mai più la stessa (Iori, 2006). Non sempre il genitore è preparato a ciò: non sempre questo genitore è sufficientemente "nato" per far nascere bene un figlio. La nascita di un figlio è pertanto evento cruciale per la vita dell'uomo e della donna: un vero e proprio spartiacque che discrimina nettamente due fasi distinte della vita: in letteratura si fa riferimento a una "transizione chiave" nella vita individuale e coniugale (Binda, 1997). Diventare genitore rappresenta all'interno del ciclo di vita una tappa fondamentale, che comporta modificazioni di rilievo, e non facili, per i membri della coppia (Zeanah, Zeananh, Stewart, 1990).

La genitorialità, dunque, non coincide sempre con la nascita di un figlio: dipende da un complesso processo di elaborazione delle relazioni affettive primarie del genitore stesso. Nadia Bruschweiler-Stern sottolinea quanto sia laborioso il percorso per la "nascita di una madre" (Stern, Bruschweiler-Stern,1998) e come il periodo perinatale sia caratterizzato da peculiari processi affettivi e cognitivi, denominati "costellazione materna" (Stern, 1995). Per la madre e il padre la nascita del figlio richiama fantasie consce e inconsce della relazione infantile con i propri genitori: la nascita del bimbo potrà consentire al genitore una riorganizzazione delle sue passate esperienze infantili, attraverso cambiamenti psicologici, sia in positivo rispetto alla rielaborazione di antiche conflittualità irrisolte che in negativo, in quanto può comportare un'alterazione dell'equilibrio della coppia in un delicato momento che è quello del passaggio dalla diade alla triade, caratterizzato da peculiari modificazioni strutturali e di investimento emotivo. Una prima rielaborazione è quella relativa al "lutto" delle proprie parti infantili verso il ruolo genitoriale, che per il genitore implica il confrontarsi con le nuove funzioni genitoriali sollecitate dalla presenza del bambino. All'interno della struttura familiare dovrà costruirsi un nuovo equilibrio che potrà riflettersi in una positiva relazione genitore-bambino, oppure al contrario potrà diventare espressione di interazioni disturbate, con particolare sofferenza per il bimbo.

Therese Benedek (1970) sottolinea che, sia per la donna sia per l'uomo, come individui e come coppia, la nascita del primo figlio rappresenti una "crisi", con un'opportunità evolutiva di maturazione. La genitorialità segna una tappa fondamentale nel processo di maturazione che si svolge nell'arco vitale dell'uomo e della donna. Nel corso di questo periodo la coppia è implicata nel compito di "salire" di una generazione, attraverso una revisione profonda di diversi tipi di relazioni interiorizzate e reali, tra cui quella con il partner, in un processo che entra a caratterizzare la qualità della relazione primaria con il bambino (Belsky, Rosenberg, Crnic, 1995). La nascita, in particolare del primo figlio, rappresenta un passaggio critico di grande complessità che richiede un riposizionamento relazionale e una ridefinizione della propria identità.

Il passaggio alla procreazione denota l'evento cruciale che demarca la separazio- 
ne tra la gioventù e l'età matura (Iori, 2006). È ciò che Erikson ha definito nel suo settimo stadio del ciclo della vita come l'antitesi critica tra generatività e la stagnazione, descrivendo la generatività come il potenziale sintonico che assorbe in sé $i$ caratteri della procreatività, della produttività e della creatività; e da qui la capacità di generare nuovi individui. La nuova "virtù" emergente da questa antitesi è la Cura, cioè una forma d'impegno in costante espansione che si esprime nel prendersi cura delle persone verso le quali se ne è contratto impegno (Erikson, 1999).

"Mettere" al mondo un figlio, come azione compiuta dai neo genitori mentre il bambino "viene" al mondo, è l'evento definito come il più naturale e al contempo più complesso (Iori, 2006). Questo atto racchiude in sé elementi di naturalità, in quanto evento biologicamente definito che appartiene alla natura umana e garantisce la prosecuzione stessa della vita e della storia dell'umanità, ma, al tempo stesso, contiene elementi di complessità in quanto vissuto ogni volta come straordinario, eccezionale, unico e irripetibile, che irrompe nell'ordinario dei giorni, modificando per sempre la fisionomia delle relazioni e delle biografie personali.

Questa svolta nell'esistenza è vissuta come frattura con un passato in cui la relazione di coppia aveva trovato modi di comunicazione definiti secondo paradigmi che ora non appaiono più adeguati. La nascita di un figlio può innescare un periodo di disorganizzazione e di turbolenza, di fronte al quale i due partner devono cercare nuove modalità di funzionamento più adeguate al cambiamento avvenuto: di fronte al nuovo possono trovare modalità di funzionamento adeguate, così come possono invece cercare modalità già sperimentate ma non adeguate, ancorché per certi versi più rassicuranti. La nascita di un figlio è evento che, lungi dall'essere relativo solo alla coppia, ha ripercussioni molto più ampie, provocando l'entrata in scena di una nuova generazione che obbliga a una ridefinizione delle relazioni familiari e a una conseguente distribuzione dei ruoli (Binda, 1997). La nascita di un bambino trasforma i componenti della coppia in genitori, ma contemporaneamente rimodella anche tutti gli altri legami di parentela. I genitori diventano nonni, i fratelli e le sorelle diventano zii e zie.

Ogni membro della famiglia si trova così a dover ricoprire contemporaneamente diversi e nuovi ruoli e a gestire una trama di relazioni sempre più ampia e variegata. La scelta di avere un figlio si intreccia così a molteplici dimensioni psicodinamiche relative alla storia della coppia, ai legami familiari e intergenerazionali, alla revisione dei propri vissuti infantili relativi alla maternità e paternità, alle differenze di genere nell'essere rispettivamente padri e madri, e in genere alla configurazione di nuove identità legate al senso di sé, alla separazione e differenziazione, e al confronto con le proprie figure genitoriali.

I due partner scelgono di solito il timing in cui concepire un figlio: ciò porta con sé un'elevata quota di novità e di imprevedibilità. L'esperienza dell'avere un bambino si accompagna a una "eccedenza", non facilmente riconducibile entro schemi precostituiti. Il figlio si inserisce nella progettualità della coppia, ma al contempo eccede tale progettualità (Scabini, Cigoli, 1991). Ogni generazione riscrive un nuovo capitolo sul rapporto tra bisogni e cura dei bisogni, e può riscriverlo in forma benigna o maligna, ma ogni generazione è anche segnata da come tale rapporto è stato istituito da chi l'ha preceduta (porta cioè in sé una relazione); l'organizzazione familiare è perciò ben più ampia e articolata della relazione genitori-figli, e la neo-coppia non è un inizio assoluto, 
ma un punto d'incontro tra due storie familiari (Scabini, 1995).

La generatività e la genitorialità, come abbiamo cercato di esplorare nel precedente capitolo, si presentano come costrutti che possono essere esplorati secondo prospettive teoriche differenti. In letteratura ritroviamo indagati da autori diversi secondo un proprio vertice di riferimento i molteplici aspetti dei processi psichici sottesi alla progettualità generativa e le processualità sottese alla gestazione. La gravidanza, concreta realizzazione del progetto stesso generativo e genitoriale, viene studiata nel suo decorso psicosomatico considerando i vissuti della donna gravida (Imbasciati, Cena, 2007): la gestazione rappresenta un evento nella vita femminile particolarmente significativo e complesso, dal punto di vista sia delle trasformazioni fisiologiche corporee che psicologiche, con la conseguente necessità di riacquistare nuovi equilibri nell'ambito della propria identità individuale e di coppia. In letteratura sono molteplici gli studi, soprattutto da parte di psicologhe e psicoanaliste donne, che hanno esplorato le diverse dimensioni connesse alle funzioni generative (Deutsch, 1945; Bibring, 1959; Benedek, 1958; Breen, 1975; Pines, 1972, 1977, 1982; Ammaniti et al., 1995; Byblowsky, 1997, 2004). Meno numerosi sono gli studi relativi a indagini nell'ambito di ciò che accade nel periodo perinatale nel padre (Lamb, 1975; Zaslow et al., 1986) e nella coppia (Mastella, 2004).

Tra gli studi in letteratura che esplorano le dimensioni connesse alle funzioni più propriamente genitoriali ci è sembrato molto rappresentativo il modello di indagine proposto da Belsky (1984), che esamina le determinanti delle differenze individuali nel funzionamento genitoriale.

Nel processo di funzionamento genitoriale del modello di Belsky sono identificati tre domini di determinanti del comportamento: personale, intendendo le risorse psicologiche dei genitori, quello delle caratteristiche del bambino e quello costituito dalle fonti sociali del lavoro e del supporto sociale. Il modello presuppone che il funzionamento dei genitori sia determinato da molteplici variabili tra loro interrelate: la personalità individuale del genitore è collegata alla relazione di coppia; intervengono poi le determinanti sociali attraverso il lavoro e le reti di supporto sociale interrelate contemporaneamente alle specificità intrapsichiche e interpersonali dei genitori; le caratteristiche del bambino hanno d'altra parte una loro peculiarità attraverso lo sviluppo fisico, psichico e relazionale, e influenzano direttamente lo sviluppo del parenting. Questo modello si è dimostrato il più collaudato in letteratura in quanto consente una comprensione olistica dei processi connessi alla genitorialità. L'idea proposta dall'autore sposta l'approccio da una visione lineare a una circolare, entro la quale i vari fattori di influenza vengono considerati e studiati sia nel loro ruolo diretto di mediatori, sia per il loro feed-back nel modificare il funzionamento di ognuno degli altri.

Il modello proposto da Belsky mostra una visione multifattoriale degli elementi che influenzano la transizione alla genitorialità, fino alla determinazione significativa dello stile genitoriale. Secondo tale modello la qualità della relazione coniugale costituisce uno dei principali sistemi di supporto per i genitori in grado di influenzare lo svolgimento della loro funzione genitoriale, nonché alcuni aspetti dello sviluppo del bambino (Belsky, 1987; Crouter et al., 1983, 1987). Nello stesso tempo la relazione coniugale appare connessa ad aspetti della storia e della per- 


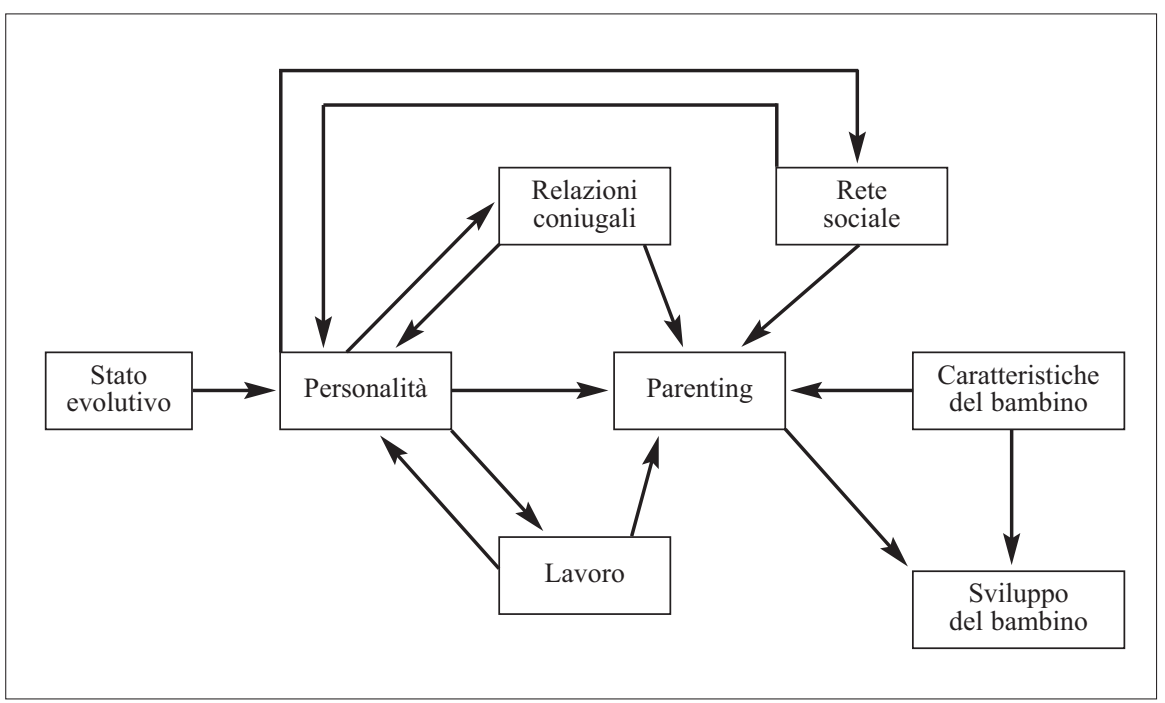

Fig. 3.1 Le determinanti del parenting: un modello del processo. La direzione delle frecce indica le reciproche e multiple connessioni dei vari fattori fra di loro e il convergere delle capacità di parenting. Modificato da Belsky, 1984

sonalità di ciascun membro della coppia, funzionando come un fattore indiretto di influenza sulla genitorialità, che può incidere sul benessere generale dei partner e costituire, fra l'altro, un predittore significativo della qualità nella negoziazione dei ruoli genitoriale (Cowan CP, Cowan PA, Shulz, Hemming, 1994) e della condivisione della responsabilità nelle cure dei figli (Simonelli, Fava Vizziello, Bighin, De Palo, Petech, 2007).

Nella prospettiva di pianificare un programma di prevenzione ${ }^{1}$ nell'ambito della salute mentale perinatale ${ }^{2}$ (capitolo 11) abbiamo ritenuto interessante e funzionale per il nostro studio il modello psicosociale proposto da Belsky (1984), da noi declinato secondo gli aspetti dinamici della Psicologia Clinica (Imbasciati, Dabrassi, Cena, 2007; 2011). Le dimensioni della generatività e della genitorialità sono state esaminate evolutivamente nell'ambito dei tre domini e considerate in interazione con le dimensioni dello sviluppo psicofisico, emotivo-affettivo, cognitivo e relazionale del bambino. Sono stati presi in considerazione anche aspetti psicosociali, rilevati attraverso i quattro compiti di sviluppo coniugali e intergenerazionali individuati nel ciclo di vita della famiglia dalla Scabini (1995). Questi possono essere

1 Progetto Interdisciplinare di Prevenzione per la tutela della Salute Mentale Perinatale "Prendersi cura dei bambini e dei loro genitori nel periodo perinatale" tra la Cattedra di Psicologia Clinica (Cena, Imbasciati, Nodari, Ziliani, Di Filippo, Doneda, Caramia) e la Cattedra di Ginecologia e Clinica Ostetrica II -Gambino Angela, del Dipartimento Materno Infantile della Facoltà di Medicina e Chirurgia dell'Università degli studi di Brescia.

2 Si ringraziano le coppie del territorio bresciano che hanno dato la loro collaborazione al progetto in corso. 
così sintetizzati: a) per quanto riguarda la relazione coniugale: ridefinire i confini della coppia e includere al suo interno gli aspetti relativi la condizione di genitorialità; b) per quanto riguarda la relazione genitoriale: assumere il ruolo genitoriale per prendersi cura della generazione più giovane, cura del figlio come elemento di dialogo intergenerazionale; c) per quanto concerne la relazione filiale (adulti come figli): superare la barriera gerarchica e ridefinire le relazioni con la famiglia d'origine, attraverso il ruolo comune di genitori; d) per quanto concerne la relazione con il contesto sociale: coordinare i tempi del lavoro con quelli della famiglia, ricorrendo all'aiuto dei servizi per l'infanzia e della famiglia estesa.

Per rilevare gli aspetti dello sviluppo psicofisico del bambino abbiamo considerato le ricerche dell'Infant Research e in particolare abbiamo fatto riferimento al "Modello dei Punti Salienti" di Brazelton, alla "Mappa della Crescita Evolutivo Funzionale" di Greenspan (Brazelton, Greenspan, 2001) e alle "Finestre Cliniche" sull'interazione genitore-bambino descritte da Stern (1995).

Il Modello dei Punti Salienti di Brazelton (2001) è stato considerato in quanto descrive i diversi periodi di sviluppo del bimbo, nei primi tre anni di vita, durante i quali i cambiamenti che i bambini compiono nel loro sviluppo portano a loro volta a dei cambiamenti all'interno del sistema familiare. I Punti Salienti rappresentano un tracciato dello sviluppo infantile per osservare il bambino stesso e l'interazione genitore-bambino. Uno strumento analogo, funzionale alla rilevazione dello sviluppo del bimbo, è considerata la Mappa della Crescita Evolutivo Funzionale di Greenspan (2001). Anche questo è uno strumento che consente di portare l'attenzione del genitore-caregiver su quelle aree evolutive nello sviluppo psicofisico ed emotivo del bimbo che è necessario monitorare attraverso un'attenta osservazione clinica, per una verifica del suo stato di benessere adeguato al periodo evolutivo del suo sviluppo psicofísico.

Abbiamo anche preso in considerazione le cinque Finestre Cliniche di Stern (1995), che vanno ad individuare, soprattutto nei primi due anni di vita del bambino, quegli "scatti" che consentono lo sviluppo di nuove capacità sociali, affettive, motorie e cognitive, che ovviamente influenzano l'interazione del bimbo con i genitori. Da 0 a 2 mesi la prima finestra clinica si riferisce a funzioni come l'alimentazione, il sonno, episodi di pianto e consolazione (Imbasciati, Cena, 2010): l'attenzione è alle interazioni che avvengono intorno a questi eventi tra genitore e bambino e che concorrono alla regolazione fisiologica ed emotiva. Dai due mesi e mezzo ai 5 mesi la seconda finestra clinica rileva aspetti della relazione primaria, tra genitore e bambino attraverso la valutazione delle interazioni faccia a faccia della diade. La terza finestra clinica dai 5 mesi e mezzo ai 9 mesi, inerente sempre all'interazione genitore-bambino, è osservabile attraverso quello che è il gioco con l'uso di oggetti. Dagli 8 ai 12 mesi la terza finestra evolutiva si riferisce agli schemi di attaccamento e all'intersoggettività (sintonizzazione degli affetti, lettura delle intenzioni dell'altro, acquisizione di un ruolo intenzionale). Dai 12 ai 18 mesi la quarta finestra evolutiva riguarda l'esplorazione del bambino nell'ambiente e la selezione delle esperienze emotive del bambino, con cui il genitore può sintonizzarsi. Dai 18 ai 24 mesi la quinta finestra evolutiva riguarda l'interazione del bambino con il genitore, la cui funzione è ora fornire aiuto, strutturare, elaborare rispo- 
ste sensibili in risposta ai desideri e alle capacità del bambino.

In una ricerca osservazionale longitudinale tutt'ora in corso abbiamo cercato di operazionalizzare, attraverso domande aperte e chiuse, aspetti sulla perinatalità individuati in letteratura (Imbasciati, Dabrassi, Cena, 2007, 2011). I quesiti relativi agli aspetti psichici della perinatalità da sottoporre alle madri e ai padri si sono rivelati, come evidenziato nei precedenti capitoli, numerosissimi, troppi per poter per ora pensare a uno strumento da validare psicometricamente, ma utili come percorso di indagine da seguire al fine di poter raccogliere, attraverso uno screening, dati rilevanti per la tutela della salute mentale perinatale e poter strutturare una progettualità di prevenzione (capitolo 11). I quesiti sulla perinatalità psichica, sono stati strutturati in una serie di questionari ${ }^{3}$ che ci consentiranno di esplorare longitudinalmente diverse aree tematiche facilmente accessibili alla soggettività cosciente della progettualità generativa e genitoriale della coppia, compresa la dimensione della sessualità, seguendo complementarmente lo sviluppo del bimbo e monitorando un periodo evolutivo della famiglia, dal concepimento-gestazione fino al terzo anno di vita del figlio.

\subsection{Fattori di rischio perinatale: sintomi di ansia, depressione, alessitimia}

Le funzioni genitoriali relative alla cura e alla protezione dei figli, quando vengono in qualche modo compromesse per esperienze negative, strutturate nella mente dei genitori lungo il loro passato, o esterne, come traumi o lutti, hanno una ricaduta sulla qualità delle interazioni primarie genitore-bambino e possono diventare fattori di rischio per il bimbo. Le esperienze che il bambino fa nel suo ambiente, le interazioni primarie, le relative rappresentazioni riguardo alle relazioni di se stesso con gli altri, costituiscono un'esperienza fondamentale per la costruzione della sua personalità, ma se le condizioni sono sfavorevoli possono anche diventare fattori di rischio di psicopatologia. I fattori di rischio sono come un insieme di variabili di tipo biologico, temperamentale, familiare, sociale, sono tra loro correlate: studi sui fattori di rischio e protezione per lo sviluppo del bambino segnalano che questi sono entrambi sempre presenti nel sistema familiare, che può diventare un contesto favorevole o sfavorevole per lo sviluppo (Candelori, Mancone, 2001).

Autorevoli studi recenti in letteratura (McHale, 2010) concordano nel rilevare nei genitori fattori di rischio quali sintomi di ansia e depressione, mancanza di sensibilità genitoriale nella relazione con il bimbo e un'adeguata rete di supporto sociale.

L'ansia è un sintomo piuttosto frequente nel periodo perinatale, a volte già in gravidanza (Grant et al., 2008) spesso associata a manifestazioni depressive (Lee et al., 2007; Faisal-Cury, Rossi Menezes, 2007; Andersson et al., 2003; Heron et al., 2004; Austin et al., 2007). I disturbi depressivi in gravidanza si ritrovano spesso associati a inadeguato sostegno sociale (Collins et al., 1993; Elsenbruck et al., 2007) o colle-

3 La Dott.ssa Cecilia Doneda, Master Genitorialità, ha partecipato alla stesura dei questionari, alla raccolta e all'inserimento dei dati per l'elaborazione statistica. 
gati a esperienze della madre di particolari eventi stressanti o di lutti (Johanson et al., 2000). I disturbi depressivi sono quelli che vengono maggiormente studiati dalla clinica perinatale (Evans et al., 2001), soprattutto nel post partum (O'Hara, Swain, 1996; Matthey, 2004), mentre quelli ansiosi, poco in evidenza nel post partum, vengono spesso considerati sovrapponibili alla problematica depressiva (Ross et al., 2003; $\mathrm{McHale}, 2010)$. In particolare si è evidenziato come manifestazioni di ansia associata a depressione (Beck, 2001; Robertson et al., 2004; Heron et al., 2004; Austin et al., 2007) possano predire un rischio maggiore di disturbi di tipo depressivo nel post partum (Matthey, 2004; Monti Agostini, 2006; Reck et al., 2008; Grant et al., 2008; Milgrom et al., 2008).

Stati elevati di ansia durante la gestazione possono comportare minacce di aborto, complicanze ostetriche durante il parto, prolungamento del travaglio, maggiore percezione dolorosa, parto distocico e nascite premature (Levey et al., 2004; Reck et al., 2010). Disturbi di ansia associati a depressione sono stati anche rilevati in relazione ad anomalie dello sviluppo fetale (Di Pietro et al., 2003; Field et al., 2003). Numerosi in letteratura sono gli studi che segnalano come elevati livelli di stress nella madre durante il periodo prenatale abbiano influenze anche sullo sviluppo del feto e del bimbo (Huizink et al., 2004; Van den Bergh et al., 2005), in particolare sullo sviluppo cognitivo, linguistico, motorio (Huinzink et al., 2003; Laplante et al., 2004; Davis, Sandman, 2010), con problematiche che possono protrarsi a lungo termine durante l'infanzia e l'adolescenza (Mennes et al., 2006; Rodriguez et al., 2005). Lo stress, (Dazzan, 2012) attraverso l'esposizione del bimbo a cortisolo, catecolamine e disfunzionamento dell'asse ipotalamo-ipofisi-surrene e del sistema simpatico-noradrenergico della madre, esercita un'influenza diretta sullo sviluppo del sistema nervoso (Buitelar et al., 2003; Ruiz Avant 2005; Austin, 2005a; Davis et al., 2007).

Alcuni studi hanno rilevato tratti temperamentali difficili nello sviluppo del bambino (Werner et al., 2007) e li hanno collegati a stati ansiosi e depressivi della madre in gravidanza: in particolare si è ritenuto che la presenza di cortisolo abbia avuto un'importante influenza sulla struttura neuromentale (temperamento) del bimbo (Davis et al., 2007), così come stati di ansia nella madre durante la gestazione siano stati collegati a disturbi emozionali e comportamentali nel bimbo (O'Connor et al., $2002,2003)$. In altre ricerche stati d'ansia e depressivi materni nel periodo prenatale sono risultati correlati al peso del bimbo (Hoffman, Hacth, 2000), a una circonferenza cranica inferiore alla nascita (Lou, Hansen, 1994), a complicanze ostetriche, a nascita pretermine (Dunkel-Schetter, 1998: Dayan et al., 2002). D'altra parte gli stati d'ansia e depressivi materni sono stati collegati a comportamenti a rischio della madre (Lindgren, 2001) e/o a rappresentazioni materne negative nei confronti del proprio ruolo materno (Hart, McMahon, 2006). L'influenza dell'ansia e della depressione materna post partum è associata a disfunzionalità nelle interazioni madrebambino (Murray-Cooper, 1999) collegate alla bassa sensibilità materna (NicolHarper et al., 2007) e alle difficoltà a esercitare il proprio ruolo di parenting (Warren et al., 2003).

Gli studi sugli stati ansiosi e depressivi paterni sono ancora pochi e in fase di evoluzione (Baldoni, Ceccarelli, 2010; Pariante, 2012): si segnalano in letteratura associazioni con le problematiche materne depressive (Misri et al., 2000) e a disturbi psichici. 
La presenza di ansia e depressione durante il periodo prenatale sono dunque un importante indicatore di rischio (Austin et al., 2005b; Edwards et al., 2008) per la salute mentale della madre, del padre e dello sviluppo del figlio e vanno tenuti in attenta valutazione per poter organizzare tempestivamente adeguati interventi preventivi.

Altro costrutto da tenere in considerazione, e peraltro da noi indagato, è "l'alessitimia”, un deficit della sensibilità emozionale che si manifesta come un'incapacità di percepire, comprendere e descrivere verbalmente i propri e gli altrui stati emotivi. Il disturbo, il cui termine fu coniato da John Nemiah e Peter Sifneos (1973) è definito come una dimensione trasversale a tutte le categorie della nosologia psichiatrica, particolarmente evidente nei pazienti psicosomatici, nei tossicodipendenti e nei pazienti affetti da Sindrome Post-Traumatica da Stress (PTSD). Successivi studi hanno mostrato come tale deficit, evidente nei casi che furono considerati patologici, sia in realtà un tratto di personalità in vari gradi e più o meno posseduto da tutti gli individui lungo un continuum; si parla pertanto dell'inverso dell'alessitimia, ovvero della capacità individuale di coscienza delle emozioni (Imbasciati, Margiotta, 2005, 2008). Vista in quest'ottica appare logico come questa capacità, cioè basso grado di alessitimia, comporti una maggiore capacità del caregiver di interagire sintonicamente col bimbo. In questo quadro l'alessitimia risulta collegata a uno stile di attaccamento insicuro evitante, con la possibilità di generare nel bimbo correlati stili di attaccamento.

Gli studi osservativi condotti su neonati nell'interazione con il loro caregiver principale (la madre), mostrano come nel bambino sia riscontrabile, fin dai primi mesi di vita, un'attività comunicativa centrata sull'espressione delle "emozioni” (RivaCrugnola, Baioni, 2002): in questo sviluppo gioca la capacità del caregiver di rispondervi adeguatamente, e dunque un basso grado di alessitimia. Lo sviluppo degli affetti e la capacità di regolazione di questi è favorito dall'esperienza di condivisione col caregiver dal "rispecchiamento" delle espressioni emotive e, in seguito, dalle interazioni giocose nelle quali si verifica l'apprendimento della capacità di individuare ed esprimere "sentimenti" (Taylor et al., 1997). In termini psicoanalitici la madre (Bion, 1962) ha un ruolo di "contenitore", per elaborare gli stati affettivi soprattutto quelli disturbanti (Taylor et al., 1997). Se questa funzione di "contenitore" e regolatore fallisce, il bambino (e poi l'adulto) sviluppa un "contenitore interno difettoso", le emozioni non sono trasformate in rappresentazioni mentali e "oggetti di pensiero", ma rimangono a livello di percezioni, sensazioni, impulsi all'azione (di qui l'alto rischio di disturbi psicosomatici) (Taylor et al., 1997).

Anche le teorie della Crittenden (1997) hanno rilevanza per il costrutto dell'alessitimia, in quanto forniscono una concettualizzazione originale dell'importanza evolutiva delle primissime relazioni di attaccamento: il soggetto impara a regolare il suo funzionamento interpersonale, e di qui quello mentale ed emotivo. Un legame di attaccamento sicuro e una buona sensibilità, responsività e "sintonizzazione" del caregiver consentono al bambino di imparare una valutazione degli affetti, propri e altrui, attraverso la cui modulazione può regolare le relazioni e arricchire così la cognizione. Oltre a sviluppare una tolleranza affettiva, il bambino impara a utilizzare i sentimenti di ansia, di depressione e gli altri affetti come segnali, che possono essere valutati e usati per scegliere un comportamento adeguato a eliminare o modifi- 
care la situazione di stress (Taylor et al., 1997). I problemi di inibizione o disregolazione affettiva nascono da stili di attaccamento insicuri: questi si associano con schemi interni e modelli di rappresentazione disposizionale che riflettono un mancato processo di integrazione delle informazioni affettive con quelle cognitive. In particolare, il bambino con attaccamento insicuro-evitante, di solito con un genitore rifiutante, emotivamente non disponibile e scarsamente espressivo, tende a sviluppare dei problemi di riconoscimento ed espressione degli affetti e impara a basarsi su di una inadeguata cognizione a stabilire relazioni. Anche il bambino con attaccamento insicuro-ambivalente, il cui genitore fornisce risposte affettive incoerenti, fuorvianti e non prevedibili, non sviluppa un'adeguata capacità di usare la cognizione e funziona sulla base di affetti non regolati.

\subsection{Fattori di protezione: sostenere la relazione genitore-bambino, la sensibilità e l'alleanza cogenitoriale}

Nel precedente volume abbiamo messo in evidenza come la Teoria dell'Attaccamento indichi la "sensibilità" genitoriale quale fattore protettivo per lo sviluppo psicofisico del bambino. In particolare abbiamo rilevato l'importanza di valutare e sostenere la relazione genitore-bambino nei modelli di prevenzione e intervento rivolti ai genitori e ai bambini nella prima infanzia.

Altro costrutto che riteniamo interessante esplorare nella nostra perinatalità è quello relativo alla cogenitorialità, presentato nel precedente capitolo. Poche sono le ricerche in letteratura che valutano la salute mentale infantile in connessione con i processi cogenitoriali (McHale, 2010).

Gli studi sulla cogenitorialità si svilupparono alla fine degli anni 80: successivamente si iniziò a pensare a strumenti idonei a valutare questo costrutto (McHale, 1995; McHale, Kuersten-Hogan, Lauretti, 2000; McHale, Kuersten-Hogan, Rao, 2004; McHale, Rotman, 2007). Sono stati costruiti diversi strumenti self-report: questionari di auto valutazione per valutare la forza della relazione cogenitoriale, setting di osservazioni naturali delle interazioni tra i membri della coppia oppure durante attività proposte, così come interviste strutturate per comprendere la natura della collaborazione del sostegno cogenitoriale. Nella maggior parte delle ricerche vengono utilizzati indici compositi che vengono costruiti combinando diverse misure di valutazione osservazionale e di rilevazione con strumenti self-report per valutare sia le interazioni coniugali intraprese da madri e padri, sia le valutazioni complessive dell'interazione coniugale e con il bimbo.

Secondo gli studi condotti da McHale (2010), è possibile prevedere quali famiglie potrebbero avere maggiori probabilità di incontrare difficoltà nel periodo dopo la nascita del bimbo attraverso una valutazione di alcuni indicatori relazionali durante il periodo prenatale: questo aspetto ci è sembrato molto utile per lo sviluppo di progetti di prevenzione e abbiamo ritenuto interessante esplorarne meglio i parametri per la progettazione di un nostro lavoro.

Il momento del periodo prenatale scelto dalla maggior parte delle ricerche 
(McHale, 2010) per la valutazione della cogenitorialità è l'inizio del terzo trimestre della gravidanza: dagli studi in letteratura emerge infatti che i genitori pensano al progetto familiare verso gli ultimi mesi della gravidanza, dal settimo al nono mese.

Studi longitudinali effettuano successive rilevazioni nelle famiglie verso la fine del primo anno di vita del bambino: questo sembra il periodo in cui inizia a comparire una diminuzione della soddisfazione coniugale, mentre è anche possibile valutare la qualità dell'attaccamento del bambino nei confronti dei genitori (Dickstein, Parke, 1988; Hirshberg, 1990). Il Progetto Family Trought Time di McHale (2010) segue la metodologia clinica di incontrare le famiglie nel terzo trimestre di gravidanza, a tre mesi dalla nascita del bambino, a 12 mesi e a 30 mesi per rilevare una stabilità dei pattern genitoriali e di adattamento del bambino.

Tale studio è per noi paradigmatico per la progettazione di ricerche perinatali e pertanto ne segnaliamo i dati più significativi e interessanti.

\subsubsection{Periodo prenatale}

Un gran numero di genitori in attesa pensa al futuro e alla nascita del bambino con una certa ansia: per alcuni si tratta di ansia per l'ignoto, per altri si tratta di ansia dovuta a preoccupazioni su come si riuscirà a essere genitori col nuovo bambino. Per alcune famiglie le preoccupazioni rispetto alla cogenitorialità si presentano insieme ad altri segnali di rischio. Nella ricerca di McHale vengono ricercati collegamenti fra quelli che potrebbero essere predittori prenatali di rischio e gli indicatori di cogenitorialità nel funzionamento della famiglia dopo il parto.

Il nucleo fondante della cogenitorialità è stato identificato da McHale (2010) nell'alleanza cogenitoriale (paragrafi 2.4-2.5), indicatore predittivo nel tempo di un adeguato adattamento e sviluppo del bimbo. Nella ricerca sperimentale "Families Through Time" è messo in evidenza come l'alleanza cogenitoriale si fondi sulle dinamiche relazionali della coppia, ancora prima della nascita del bambino. La qualità delle dinamiche relazionali prenatali predirebbe il funzionamento cogenitoriale precoce: questa prospettiva focalizza l'attenzione nei programmi preventivi prenatali sulla consultazione della coppia, di cui dovrebbe essere presa in attenta considerazione la storia dei problemi relazionali e della qualità della comunicazione.

Nelle coppie che sperimentano tensioni coniugali, in cui i genitori sono pessimisti sulle proprie capacità di alleanza genitoriale, esiste una maggiore possibilità che il processo genitoriale sia caratterizzato da bassi livelli di solidarietà e sostegno genitoriale. La capacità di coping dei genitori è provata con la nascita del bambino e gli interventi prenatali devono essere focalizzati ad aiutare i genitori a costruire un'autoconsapevolezza individuale per rafforzare queste capacità.

I dati delle ricerche concordano nel rilevare come le coppie che hanno avuto delle difficoltà prima della nascita del bambino e che hanno espresso preoccupazioni sulla capacità di costruire una relazione positiva con il partner, mostrino livelli più bassi di coesione e sostegno reciproco. Tra gli indicatori di funzionamento familiare vengono rilevati la soddisfazione coniugale percepita rispetto alla divisione dei compiti di cura del bambino e la collaborazione e solidarietà tra i genitori. 
Indicatori prenatali di rischio, segnalati nella ricerca di McHale (2010) riguardano prevalentemente sintomatologie depressive, scarso autocontrollo, bassa resilienza, disagio coniugale, attaccamento insicuro, previsioni negative sulla famiglia futura.

Gli studi che prendono in considerazione il disagio coniugale nella transizione alla genitorialità (Cowan, Cowan, 1992; Lewis, 1989; McHale, 2010) sono focalizzati sulla conoscenza del funzionamento relazionale dei partner durante la gravidanza, attraverso una valutazione del loro stato emotivo, una conoscenza delle rappresentazioni delle loro relazioni intime e dei loro legami di attaccamento. Gli indicatori presi in considerazione sono la qualità della relazione coniugale, le caratteristiche di personalità dei genitori, i legami di attaccamento, le aspettative dei genitori rispetto alla vita familiare dopo la nascita del bambino. Ognuno di questi fattori viene considerato come un potenziale precursore dell'adattamento successivo della coppia genitoriale nei primi mesi dopo il parto.

Le rilevazioni in gravidanza segnalano come i genitori considerino affidabili le relazioni interpersonali per una collaborazione cogenitoriale e basino le loro interazioni sul coordinamento, l'intimità e la reciprocità attraverso relazioni rispettose e supportive per il coniuge. Le relazioni di attaccamento sicure nei padri e nelle madri sono un fattore protettivo per la coppia perché favoriscono la possibilità di costruire un'alleanza cogenitoriale supportiva (Talbot, Elliston, Thompson et al., 2006).

Le anticipazioni dei genitori sulla loro vita familiare futura sono prevalentemente legate alle loro passate esperienze: rilievo ha la considerazione della cogenitorialità nella famiglia di origine (McHale, Kazali, Rotman et al., 2004). Le storie familiari variano rispetto agli stili di attaccamento: genitori con attaccamento sicuro nei confronti dei propri genitori rispondono in maniera sensibile e contingente ai bisogni dei bambini. Stili di attaccamento insicuro hanno maggiore probabilità di disturbare la capacità di sintonizzarsi col bambino e per soddisfare correttamente i suoi bisogni, dando origine ad attaccamenti infantili insicuri.

Le valutazioni dell'attaccamento attraverso l'Adult Attachment Interview, durante il terzo trimestre di gravidanza, consentono di predire la qualità della relazione cogenitoriale dopo il parto (McHale, 2010) e la valutazione delle rappresentazioni generalizzate di attaccamento degli adulti. La qualità del legame di attaccamento del genitore nei confronti del proprio genitore consente di predire la qualità del legame di attaccamento del bambino, e consente anche di sviluppare dei modelli operativi relativamente all'essere genitori e alle dinamiche familiari. I genitori che provengono da famiglie con grave disagio cogenitoriale sono maggiormente esposte a sperimentare maggiori difficoltà nella loro cogenitorialità.

I bassi livelli di coerenza nello stato della mente dei genitori predicono aspetti negativi (De Luccie, 1995) della cogenitorialità. Gli interventi preventivi devono focalizzarsi sulla capacità della coppia a sintonizzarsi con le proprie intense emozio$\mathrm{ni}$, molte non espresse o inibite, orientando i genitori a cercare un linguaggio comune per esprimere i propri sentimenti, soprattutto negativi, imparando in tal modo modalità di autocontrollo nei momenti di maggiore conflittualità. I genitori desiderano fare e dare il meglio ai propri figli: spesso però succede che si intraprendano percorsi diversi da quelli desiderati, e può accadere che i genitori operino con scopi contrastanti, anche se individualmente il loro impegno è orientato a fare il meglio per il 
proprio bambino. Per tali ragioni gli interventi preventivi orientati ad aiutare le coppie a prepararsi alla nascita del proprio bambino potranno sviluppare effetti a lungo termine solo se saranno seguiti da interventi post natali.

Le aspettative prenatali negative, altro indicatore rispetto alla futura cogenitorialità, possono influenzare i primi sforzi cogenitoriali: aspettative e previsioni riguardano l'accudimento del bambino, cioè le responsabilità che ognuno dei genitori si assumerà nel suo accudimento. Le ricerche mettono in evidenza che i problemi sorgono quando vengono disattese le aspettative prenatali delle madri sulla divisione con i padri delle responsabilità nella cura del bambino. Una differenza di punti di vista sulla genitorialità tra madri e padri è un importante indicatore di futuri problemi, così come una divergenza di idee sulla genitorialità dei due partner. Molta importanza ha anche la valutazione delle discrepanze fra aspettative vissute come ideali e quelle ritenute realistiche: tale grado di discrepanza può essere valutato. Le aspettative sulla suddivisione attesa nei compiti e responsabilità familiari sono anch'esse indicatori di rischio. I Cowan (Cowan, Cowan, 1988) hanno messo in evidenza la differenza tra la divisione di compiti e responsabilità all'arrivo del bambino e quella ideale: il divario, fra come sarà e come i genitori avrebbero voluto che fosse, indica la dimensione delle preoccupazioni rispetto alla cogenitorialità. Le ricerche hanno messo in evidenza come le donne, ma anche gli uomini stessi, si aspettino che i padri facciano più di quello che effettivamente finiscono col fare (Cowan, Cowan, 1992) e quando queste aspettative non vengono rispettate le madri manifestano maggiori difficoltà di adattamento alla genitorialità, sperimentando una forte delusione nel matrimonio. Numerose ricerche hanno affrontato gli adattamenti delle coppie alla prima genitorialità (Cowan, Cowan, 1992; Entwisle, Doering, 1981; Lewis, 1989) e c'è concordanza nell'evidenziare una diminuzione della soddisfazione coniugale.

\subsubsection{Tre mesi dopo la nascita del bimbo}

Si ritiene che in quest'epoca le coppie siano riuscite a costruire i propri peculiari pattern cogenitoriali e un proprio stile di rapporto. Si valuta così l'adattamento genitoriale individuale e coniugale, l'alleanza cogenitoriale, le relazioni genitore-bambino e la capacità di auto regolazione del bambino. I genitori che non riescono ancora a lavorare in modo cooperativo avranno maggiori probabilità di sperimentare di nuovo in questo periodo la loro difficile situazione. Una nascente alleanza cogenitoriale può essere compromessa da sintomatologia depressiva, insoddisfazione coniugale, inadeguata divisione del lavoro nella cura del bambino. Cambiamenti individuali e relazionali sono spesso contrassegnati da un elevato tasso di depressione e insoddisfazione coniugale A tre mesi dal parto viene individuato il declino più evidente della soddisfazione coniugale (Belsky, Spanier, Rovine, 1983): alcuni autori rilevano che le maggiori affermazioni di soddisfazione vengono espresse dalle madri dal $1^{\circ}$ al $3^{\circ}$ mese dopo il parto (Fleming et al., 1990), mentre dopo iniziano ad esprimere sentimenti meno positivi, in special modo verso il marito. In questo periodo il bambino ha sul sistema familiare un impatto maggiore, per lo sviluppo delle prime capacità di interazione e regolazione e per il maggiore coordinamento at- 
tentivo con una condivisione affettiva spesso diversa con ognuno dei due genitori. Le coliche che quasi sempre si verificano verso il $3^{\circ}$ mese del bimbo, in concomitanza con altri disturbi del sonno e della alimentazione, possono sollecitare stati di maggiore stress nei genitori.

Valutazioni che indicano l'adattamento genitoriale sono quelle relative al racconto della nascita. Le madri che presentano una sintomatologia depressiva elevata non concordano con i punti di vista dei partner. Per contro nelle famiglie in cui la madre disconferma il padre, questi riferisce un'insoddisfazione coniugale maggiore. L'insoddisfazione coniugale e la depressione a tre mesi sono amplificate in quelle donne le cui aspettative coniugali circa la divisione delle responsabilità di accudimento del bambino da parte del partner sono state disattese.

I dati della ricerca di McHale (2010) indicano che le aspettative prenatali dei genitori prefigurano la solidarietà genitoriale: genitori con aspettative negative presentano i livelli più bassi di coesione genitoriale quando il bambino ha tre mesi. Più specificatamente sarebbero le aspettative negative della madre, più che quelle del padre, a predire un ritiro del padre dalla solidarietà genitoriale (McHale, 2010).

Manifestazioni di pessimismo durante la gravidanza sembrano adombrare la relativa mancanza di un alleanza coordinata che consenta di offrire conferma reciproca: il pessimismo della donna rispetto alla solidarietà genitoriale durante la gravidanza più che il pessimismo dell'uomo predice una maggiore disconnessione da parte dell'uomo durante le negoziazioni cogenitoriali. Il pessimismo prenatale non predice tuttavia un elevato conflitto cogenitoriale: le coppie in cui un partner mette in dubbio la propria capacità di collaborare come genitore mettono in evidenza come i partner stiano cercando di trovare dei modi per lavorare insieme. Una aumentata sensibilità alle difficoltà di lavorare può essere anche ciò che può averli tenuti a distanza per evitare lo sviluppo di una relazione conflittuale, ma non prefigura necessariamente conflittualità. Le madri e i padri che esprimono preoccupazioni su come loro e il partner potranno collaborare nel post partum non hanno una probabilità maggiore di comportarsi in modo squalificante con i partner.

Il conflitto cogenitoriale è predetto da un fattore prenatale: la coerenza dello stato della mente riguardo all'attaccamento della donna. Le madri che hanno rivelato incoerenza nella valutazione della AAI, manifestano con maggiori probabilità conflittualità cogenitoriali. L'insicurezza materna deve essere valutata attentamente durante il periodo prenatale e considerata come un fattore potenziale di rischio per manifestazione di conflitti cogenitoriali (Talbot, Elliston, Thompson, et al., 2006). I padri che mostrano uno stato della mente meno coerente rispetto all'attaccamento durante il periodo prenatale non hanno invece maggiori probabilità di venire coinvolti in conflitti cogenitoriali, ma presentano maggiori comportamenti di ritiro.

\subsubsection{II primo anno di vita}

È questo il periodo in cui il bimbo sviluppa le prime forme di intersoggettività (Stern, 1985), ricerca un'attenzione condivisa nei giochi, sviluppa un legame di 
attaccamento (Ainsworth et al., 1978) e inizia a rispondere ai conflitti fra adulti (DeJonghe, Bogart, Levendosky, 2005; Hirshberg, 1990; Zaslow et al., 1989).

L'alleanza cogenitoriale al primo compleanno del bambino sarà funzionale agli stress che i genitori hanno sperimentato e superato dopo la nascita (McHale, 2010): la lamentela più comune delle madri è il frequente disimpegno dei padri, che risulta maggiore di quello che le madri prevedevano. La convinzione delle madri è prevalentemente quella che il padre non stia facendo la sua parte nel prendersi cura del bambino: se queste percezioni sono già presenti a tre mesi e non vengono adeguatamente affrontate, si ritroveranno amplificate più in là nel periodo perinatale. I padri a loro volta hanno la percezione di dedicare il loro tempo alla famiglia a discapito di altri impegni, e di non essere adeguatamente apprezzati. Un grande rischio è che i padri e le madri non si sentano convalidati dal proprio partner.

Nella solidarietà genitoriale valutata attraverso la metodologia dell'intervista le madri in genere parlano a lungo delle proprie emozioni e spesso parlano di che cosa vorrebbero cambiare nel partner: sono preoccupate della regolarità con cui il padre si prende cura del bambino, alcune cercano più aiuto, altre si aspettano che il padre accudisca il bambino senza che loro glielo chiedano. Le preoccupazioni del padre riguardano invece prevalentemente l'organizzazione e la strutturazione della giornata del bambino, rispetto soprattutto alla quantità di tempo passato insieme e al lavoro extra domestico della madre. Le madri esprimono poco la preoccupazione dell'ambiente di apprendimento offerta al bambino rispetto al bisogno di ridurre gli impegni del lavoro: i cambiamenti desiderati sono quelli di passare comunque più tempo insieme, come famiglia, e di dedicare più tempo al figlio.

I padri sottolineano spesso le differenze di stile educativo rispetto alla partner, mentre le madri esprimono preoccupazioni relative alla mancanza di sostegno da parte del padre ed esprimono la propria frustrazione nel dover far rispettare le regole rispetto al poco tempo e la scarsa energia con cui il padre si dedica alla famiglia.

Una dinamica genitoriale frequente nelle famiglie con il bambino di un anno è quella in cui il padre fa affidamento sulla madre e la considera pressoché totale responsabile, limitandosi a esprimere le proprie preoccupazioni quando si accorge che il clima è troppo severo. La madre cerca una partecipazione maggiore da parte del padre, ma raramente la coppia è in questo caso soddisfatta del clima familiare che sta creando. In primo piano vi è la necessità di dedicare più tempo alla famiglia: a tre mesi le madri iniziano a dire che si sentono frustrate perché devono prendersi cura del bambino con modalità che la coppia non aveva immaginato e la discussione tra genitori avviene sulla divisione dei compiti; a 12 mesi questi sono abbastanza definiti e le madri parlano apertamente delle proprie frustrazioni, del desiderio di avere più tempo per sé, e della frustrazione quando vedono che il padre viene accolto e ascoltato dal bambino anche se si occupa meno di lui. Durante la gravidanza pochi genitori sono riusciti a prevedere quale sarebbe stata la vita familiare dopo la nascita del bambino. Un disagio coniugale compromette ovviamente la relazione genitore bambino (Erel, Burman, 1995): la coesione della dinamica relazionale dei genitori predice la qualità dell'interazione genitore-bambino (McHale, Lauretti, Talbot, 2002). 


\subsubsection{Trenta mesi di età del bambino}

Una terza rilevazione in cui si valuta la continuità e i cambiamenti della cogenitorialità, viene effettuata a metà del terzo anno di vita del bambino, a 30 mesi dopo la nascita. I due anni sono anche un momento importante nello sviluppo del bambino per le opposizioni che i figli esprimono ai genitori con il loro "no", nonché per l'educazione sfinterica e le regole che iniziano a venire stabilite. È l'epoca in cui si manifestano i problemi che si etichettano come "capricci". In questo periodo dello sviluppo è necessario che i genitori bilancino i bisogni di protettività e di autonomia del bimbo. Durante il terzo anno di vita dei bambini i genitori cercano di educarli al rispetto delle regole e ad assumere le buone abitudini: se vi è disaccordo si inviano messaggi differenti e i bambini entrano in uno stato di confusione. Il disaccordo fra i partner confonde e disorganizza i figli e crea coalizioni genitore- figlio distruttive per la famiglia (Minuchin, 1974). Questo periodo sembra cruciale per le relazioni genitore-bambino. La maggiore autonomia del bimbo comporta uno sforzo maggiore da parte dei genitori nel far rispettare le regole: le coppie con scarsa solidarietà cogenitoriale, in cui uno dei due partner si mette da parte durante gli episodi di conflitto con il bimbo, priva il figlio della possibilità di avere un aiuto sicuro per regolare le emozioni e gli fornisce contemporaneamente modelli di indisponibilità emozionale.

Un costrutto che ha suscitato interesse riguarda una capacità flessibile e resistente identificata nella "resilienza" (Block, Block, 1980). Con tale termine si intende la capacità di conservare buone disposizioni d'animo e buone capacità genitoriali, peraltro già dimostrate, anche quando circostanze avverse e conflitti coniugali ne avrebbero fatto prevedere un calo o un crollo. La resilienza sembra essere una risorsa particolare dei padri.

La principale verifica delle indagini a trenta mesi è la continuità nel tempo dell'adattamento cogenitoriale e del bambino: sembra che le coppie che presentano minori indici di rischio durante la gravidanza mostrino una maggiore solidarietà cogenitoriale a tre, dodici e trenta mesi di vita del bambino. La tensione cogenitoriale sembra si manifesti più frequentemente nei primi mesi, mentre i modelli cogenitoriali più stabili sarebbero stati osservati a 12 mesi e una maggiore capacità di adattamento a 30 mesi.

Nel bimbo vengono valutati problemi comportamentali, capacità adattative, competenze scolastiche e sociali, comprensione delle emozioni, capacità di gioco: i diversi indicatori di adattamento infantile possono essere sommati per formare un indicatore universale di adattamento o disadattamento. Una buona cogenitorialità può aiutare a plasmare i modelli di adattamento del bambino. Le famiglie in cui è presente maggiore coesione, calore, conferma, minore antagonismo e minor disimpegno proseguono in genere su percorsi adattativi fino a 30 mesi.

Uno dei compiti più impegnativi per i genitori di bambini di 30 mesi è quello di aiutarli a controllare e integrare le proprie emozioni. I bambini possono ricorrere a diverse strategie di regolazione emozionale per soddisfare le differenti esigenze dei genitori. Nelle famiglie in cui le madri si impegnano poco nell'addestramento emozionale dei bambini, questi mostrano livelli più alti di espressività negativa, soprat- 
tutto se il padre manifesta poco impegno. Nelle famiglie in cui il padre si impegna in attività di addestramento emozionale, i bambini a 30 mesi esprimono meno emozioni negative (DeCourcey, 2005). Anche se i genitori vedono le emozioni in modo diverso, tuttavia, se conducono un lavoro congiunto, possono evitare che queste differenze coinvolgano il bambino. Le alleanze realmente funzionanti sono quelle in cui i due partner sono consapevoli di quello che ognuno di loro pensa, rispetto a ciò che deve fare l'altro, anche se non lo condivide pienamente.

\subsection{La nascita del secondo figlio}

Con la nascita di un secondo bambino vengono a modificarsi le dinamiche familiari e si ha una riorganizzazione della dinamica del gruppo. La ricerca di McHale (2010) permette di confrontare la cogenitorialità nelle famiglie con un figlio piuttosto che con due.

I genitori con un solo figlio rispetto ai genitori con due figli parlano della propria vita in modo differente: la principale differenza sta nel grado maggiore di tonalità affettiva sottesa al racconto dei due genitori. I racconti dei padri e delle madri con un figlio di un anno presentano tonalità affettive più positive di quelli dei due anni: le madri descrivono con maggiore facilità momenti di riunione familiare, mentre con due figli i genitori ricorrono più facilmente al racconto dei momenti peggiori e le madri tendono a considerare rari i momenti belli. Questo è indicativo che i genitori con due bambini vivano in un clima meno positivo per i propri figli. Il primo figlio assorbe una quantità maggiore di energia dei genitori e li coinvolge maggiormente dal punto di vista emotivo: la prima esperienza di genitorialità è per loro una scoperta che cattura maggiormente la loro attenzione. Con due figli i genitori acquistano maggiore esperienza e questo comporta una visione più realistica e pacata dei momenti difficili della vita familiare, con tonalità affettive più moderate.

Le madri con due figli raccontano prevalentemente avvenimenti accaduti fuori dal contesto familiare, tendenza questa che non si riscontra nelle madri con un figlio unico: un'ipotesi prospettata è che le famiglie con due figli tendono ad avere una vita sociale più allargata e che i momenti migliori possono verificarsi fuori casa.

La nascita del primo figlio disorganizza le relazioni di coppia in maniera più radicale rispetto alla nascita del secondo figlio: i genitori alla nascita del secondo figlio sanno cosa aspettarsi. La genitorialità con il primo figlio dovrebbe presentarsi come meno organizzata, ma anche l'arrivo del secondo comporta una riorganizzazione importante.

Scarse ricerche hanno studiato la diversità del comportamento genitoriale verso due diversi fratelli all'interno dello stesso sistema familiare. Le ricerche hanno sostanzialmente confermato quello che le famiglie già sanno (Kreppner, 1988; Kreppner, Paulsen, Schuetze, 1982): quando i secondi figli crescono e partecipano alle dinamiche familiari, uno dei principali compiti dei genitori è quella del controllo della rivalità fraterna. Attraverso l'osservazione delle interazioni di gioco familiare (Coparenting Family Rating System, McHale, Kuersten-Hogan, Lauretti, 
2000), vengono valutati indici di sostegno, coesione, antagonismo, coinvolgimento e viene studiato il modo in cui ognuno dei due adulti si comporta con i due bambini: viene valutata la qualità della loro cooperazione e la diversità dell'impegno, connesso con gli indici che valutano la presenza di competizione e di disaccordo genitoriale. La competizione viene valutata rispetto al modo in cui i genitori si mettono reciprocamente in rivalità per ottenere l'attenzione e l'affetto del figlio.

I processi genitoriali variano in funzione dell'ordine di nascita del bambino: sembra che questo influenzi la qualità della genitorialità. Il comportamento dei genitori con il primo figlio è molto diverso dal comportamento con il secondo: la coppia genitoriale è più coinvolta e attenta quando interagisce con il primo figlio, mentre i secondi ricevono un impegno meno concordato e coordinato. I genitori mostrano nei confronti del secondo figlio un'attenzione congiunta meno intensa di quella che hanno prestato al primo figlio. Vengono anche rilevate differenze nelle modalità di accudimento: i genitori manifestano un investimento congiunto più intenso nel prendersi cura del bambino più grande; il padre manifesta una maggiore responsabilità con il figlio maggiore, mentre la madre si prende la responsabilità principale del bambino più piccolo. Questi dati sono molto evidenti quando i due bambini hanno una piccola differenza di età, mentre le cose cambiano con due figli, in cui uno ha un anno e l'altro è in età prescolare.

I dati sui processi genitoriali osservati variano dunque in base alla posizione ordinale del bambino nella famiglia: il confronto della cura del primo bambino di 12 mesi, rispetto alla cura del secondo bambino di 12 mesi mette in evidenza livelli più alti di investimento congiunto dei genitori con il primo bambino. Questi risultati confermano quelli rilevati da precedenti ricerche (Belsky, Gilstrap, Rovine, 1984). Un'ipotesi è che l'esperienza con il primo figlio abbia abbassato il livello di ansia, di preoccupazione e di permissività nei confronti del secondo figlio. La differenza non sembra invece riferibile alle dimensioni dell'armonia della coppia piuttosto che della dissonanza. La differenza è riferita alla quantità e intensità dell'investimento congiunto, non alla qualità della coordinazione genitoriale.

La ricerca scientifica conferma ciò che viene rilevato dall'esperienza comune: i genitori non trattano i figli tutti nello stesso modo: cooperano maggiormente quando si occupano del primo figlio con più direttive, maggiormente controllate e strutturate. Altre ricerche mettono in evidenza come i genitori diano maggiore importanza alla disciplina, al controllo e ad attività strutturate con i primi figli (Baydar, Geek, Brocks-Gunn, 1997; Volling, Elins 1998): i genitori rispondono alle diverse esigenze di sviluppo dei figli con un maggiore controllo sui primi figli, adeguandosi alle esigenze del loro sviluppo fisico e cognitivo.

\section{Bibliografia}

Ainsworth MS, Blehar, MC, Waters, E, Wall S (1978) Patterns of attachment: a psychological study of the strange situation (vol. XVIII). Potomac. Erlbaum, Lawrence, MD

Ammaniti M, Candelori C, Pola M, Tambelli R (1995) Maternità e gravidanza: Studio delle rappresentazioni materne. Cortina, Milano 
Andersson L, Sundering-Poroma I, Bixo M, Wulff M, Bondestam K, LåStröm M (2003). Point prevalence of psychiatric disorders during the second trimester of pregnancy: a populationbased study. American Journal of Obstetrician Gynecology 189 1:148-154

Austin MP, Leader LR, Reilly N (2005a) Prenatal stress, the hypothalamic-pituitary-adrenal axis, and fetal and infant neuro behavior. Early Human Development 81:917-926

Austin MP, Hazdi-Pavlovic D, Saint K, Parker G (2005b) Antenatal screening for the prediction of postnatal depression: validation of a psychosocial Pregnancy Risk Questionnaire. Acta Psychiatrica Scandinavica 112 4:310-317

Austin MP, Tully L, Parker G. (2007) Examining the relationship between antenatal anxiety and postnatal depression. Journal of Affective. Disorders 101:169-174

Baldoni F, Ceccarelli L (2010) La depressione perinatale paterna. Una rassegna della ricerca clinica ed empirica. Infanzia e Adolescenza 9:79-92

Baydar N, Geek A, Brocks-Gunn J (1997) A longitudinal study of the effects of the birth of a siblings during the first 6 years of live. Journal of Marriage and the Family 59:939-956

Belsky J, Gilstrap B, Rovine M (1984) The Pensylvania infant and family development project: stability and change in mother-infant and father-infant interaction in a family setting at one, free and nine mounth. Child Development 55:692-705

Belsky J, Spanier GB, Rovine M (1983) Stability and change in marriage across the transition to parenthood. Journal of Marriage and the Family 45:567-577

Belsky J (1984) The determinants of parenting: a process model. Child Development 55:83-96

Belsky J (1987) Early human experience: A family perspective. Development Psychology 17:3-23

Belsky J, Rosenberg K, Crnic K (1995) Maternal personality, material quality, social support and infant temperament: Their significance for mother-infant attachment in human families. In: Pryce C, Martin R, Skuse D (a cura di) Motherhood in human and nonhuman primates: Prosocial determinants. Plenum Press, New York, pp 115-124

Beck CT (2001) Predictors of postnatal depression: an update. Nursery Research 50:275-285

Benedek T (1958) Parenthood as a development phase: a contribution to the libido theory. Journal of the American Psychoanalytic Association 7:389-417

Benedek T (1970) Parenthood during the life cicle. In: Anthony EJ, Benedek T (a cura di), Parenthood: Its psychology and psychopathology. Little Brown \& Co., Boston

Bibring GL (1959) Some considerations of the psychological process in pregnancy. The Psychoanalytic Study of the Child 16:113-121

Binda W (1997) Diventare famiglia. Angeli, Milano, 1997

Bion WR (1962) Learning from experience. Heinemann, London. Tr.it. Apprendere dall'esperienza. Armando, Roma, 1972

Block JH, Block J (1980) The role of ego control and ego resiliency in the organization of behavior In Collins WA (a cura di) Minnesota Symposium on Child Psychology, 13. Earlbaub, Hillsdale, pp 39-109

Brazelton TB (2001) Il modello dei punti salienti. In Brazelton TB, Greenspan SI. I Bisogni irrinunciabili dei bambini. Cortina, Milano

Brazelton TB, Greespan S (2001) I bisogni irrinunciabili dei bambini: ciò che un bambino deve avere per crescere e imparare. Cortina, Milano, 2001

Breen D (1975) The born of the first child. Tavistock, London

Buitelaar JK, Huizink AC, Mulder EJ, de Medina PG, Visser GH (2003) Prenatal stress and cognitive Development and temperament in infants. Neurobiology of Aging 24:53-60

Bydlowski M (1997) Il debito di vita. Itinerario psicoanalitico della maternità. Quattroventi, Urbino

Bydlowski M (2004) Sognare un figlio. L'esperienza interiore della maternità. Pendragon, Bologna

Candelori C, Mancone A (2001) Genitorialità: situazioni a rischio e psicopatologiche. Cortina, Milano

Capodieci S, Ferraro I, Dell'alba B, Ruolo G, Baldo M, Canu B (1990) Gravidanza, maternità, paternità e vita sessuale di coppia. Rivista di sessuologia 14:331-346

Chase-Landsale PL, Owen MT (1987) Maternal employment in a family context Effects on infant 
mother and infant-father attachments. Child Development 58:1505-1512

Collins NL, Dunkel-Schetter C, Lobel M, Scrimshaw SC (1993) Social support in pregnancy: psychosocial correlates of birth outcomes and postpartum depression. Journal Pers Soc Psychol 65:1243-1258

Cowan CP, Cowan PA (1988) Who does what when partner become parents: implications for men, woman and marriage. Marriage and Family review 12:105-131

Cowan CP, Cowan PA (1992) Dall'alcova al nido. La crisi della coppia alla nascita di un figlio. Cortina, Milano

Cowan CP, Cowan PA, Shulz PA, Hemming G (1994) Preschool family factors in children's adaptation to kinder-garden. In: Parke R, Kellam S (a cura di), Exploring family relation-ships with other social contexts: Advances in family research, 4. Earlbaum, Hillsdale

Crittenden PM (1997) Pericolo, sviluppo e adattamento. Masson, Milano

Crouter A, Belsky J, Spanier GB (1983) The family context of child development. In G. Whitehurst (a cura di). Annals of child development, 1, Greenwich, JAI

Crouter AC, Perry-Jenkins M, Huston TL, McHale SM (1987) Processes underlying father involvement in dual-earner and single-earner families. Developmental Psychology 23:431-440

Davis EP, Glynn LM, Schetter CD, Hobel C, Chicz-Demet A, Sandman CA (2007) Prenatal exposure to maternal depression and cortisol influences infant temperament 46:737-746

Davis EP, Sandman CA (2010) The timing of prenatal exposure to maternal cortisol and psychosocial stress is associated with human infant cognitive development. Child Development 81:131-48

Dayan J, Creveuil C, Herlicoviez M, Herbel C, Baranger E, Savoye C, Thouin A (2002) Role of anxiety and depression in the onset of spontaneous preterm labor. American Journal of Epidemiology 155:293-301

Dazzan P (2012) Fattori biologici di rischio per la psicosi post-partum. Seminario "Depressione e psicosi post-partum: fattori di rischio". Facoltà di medicina e chirurgia, Università degli Studi di Brescia, 26 marzo 2012 (in stampa)

De Luccie MF (1995) Mothers as gatekeepers: A model of maternal mediators of father involvement. Journal of Genetic Psychology 152:225-238

De Renoche I, Della Giustina L (1989) Ambivalenze e conflittualità nel divenire madre. Bambino incompiuto - IPSSER. Anno 6:73-80

DeCourcey WM, Haskell V, McHale JP (2005) Concordance of Coparents' Meta-EmotionStrategies and Toddler emotion expression. Relazione presentata alla Society of Research in Child Development, Atlanta, GA

DeJonghe ES, Bogart GA, Levendosky AA (2005) Infant exposure to domestic violence predicts heightened sensitivity to adult verbal conflict. Infant Mental Health Journal 26:268-281

Deutsch H (1945) Psicologia della donna. Vol. I (1945) Vol. II (1946), Boringhieri, Torino, 1971

Dickstein S, Parke RD (1988) Social referencing in infancy: A glance at fathers and marriage. Child Development 59:507-511

Di Pietro JA, Costigan KA, Gurewitsch ED (2003). Fetal response to induced maternal stress. Early Human Development 74:125-138

Dunkel-Schetter C (1998) Maternal stress and preterm delivery. Prenatal and Neonatal medicine: the International Journal of basic and clinical research and practice 3:39-42

Edwards B, Galletly C, Semmler-Booth T, Dekker G (2008) Does antenatal screening for psychosocial risk factors predict postnatal depression? A follow-up study of 154 women in Adelaide, South Australia. Australian and New Zeland Journal of Psychiatry 42:51-55

Elsenbruch S, Benson S, Rucke M, Rose M, Dudenhausen J, Pincus-Knackstedt MK, Klapp BF, Arck PC (2007) Social support during pregnancy: effects on maternal depressive symptoms, smoking and pregnancy outcome. Human Reproduction 22:869-877

Entwisle D, Doering S (1981) The first Birth: a turning point. Johns Hopkins University Press, Baltimore

Erel O, Burman B (1995) Interrelatedness of marital relations and parent-child relations: A meta analitic review. Psychological Bullettin 118:108-132

Erikson HE (1999) I cicli della vita. Continuità e mutamenti, Armando, Roma 
Evans J, Heron J, Francomb H, Oke S, Golding J (2001) Cohort study of depressed mood during pregnancy and after childbirth. British Medical Journal 3123:257-260

Faisal-Cury A, Rossi Menezes P (2007) Prevalence of anxiety and depression during pregnancy in a private setting sample. University Hospital, Section of Epidemiology, University of São Paulo, São Paulo, Brazil

Field T, Diego M, Hernandez-Reif M, Schanberg S, Kuhn C, Yando R (2003). Pregnancy anxiety and comorbid depression and anger effects on the fetus and the neonate. Depression and Anxiety 17:140-151

Fleming AS, Ruble DN, Flett GL, Van Wagner V (1990) Adjustment in first time mothers: changes in mood and mood content during the early post partum month. Developmental Psychology 26:137-143

Grant KA, McMahon C, Austin MP (2008). Maternal anxiety during the transition to parenthood: A prospective study. Journal of Affective Disorders 108:101-111

Greespan S (2001) Mappa e questionario della crescita evolutivo-funzionale. In: Brazelton TB, Greespan S (a cura di) I bisogni irrinunciabili dei bambini: ciò che un bambino deve avere per crescere e imparare. Cortina, Milano, 2001

Hart R, McMahon CA (2006) Mood state and psychological adjustment to pregnancy. Arch Women Ment Health 9:329-337

Heron J, O'Connor TG, Evans J, Golding J, Glover V, ALSPAC study team (2004) The course of anxiety and depression through pregnancy and the postpartum in a community sample. Journal of Affective Disorders 80:65-73

Hirshberg LM (1990) When infant look to their parents: Twelve month-olds response to conflicting parental emotional signals. Child Development 61:1187-1191

Hoffman, S, Hatch, MC (2000) Depressive symptomatology during pregnancy: Evidence for an association with decreased fetal growth in pregnancies of lower social class women. Health Psychology: Official Journal of the Division of Health American Psychology Association 19:535-543

Huizink AC, Robles de Medina PG, Mulder EJH, Visser GHA, Buitelaar JK (2003) Stress during pregnancy is associated with developmental outcome in infancy. Journal of Child Psychology and Psychiatry 44:810-818

Huizink AC, Mulder EJ, Buitelaar JK (2004) Prenatal stress and risk for psychopathology: specific effects or induction of general susceptibility? Psychological Bulletin 130:115-142

Imbasciati A, Cena L (2007) Psicosomatica della gravidanza e del parto. In: Imbasciati A, Dabrassi F, Cena L (a cura di) Psicologia Clinica Perinatale. Piccin, Padova

Imbasciati A, Cena L (2010) I bambini e i loro caregiver. Borla, Roma

Imbasciati A, Dabrassi F, Cena L (2007). Psicologia Clinica Perinatale. Piccin, Padova

Imbasciati A, Dabrassi F, Cena L (2011) Psicologia Clinica Perinatale per lo sviluppo del futuro individuo. Un uomo transgenerazionale. Espress, Torino

Imbasciati A, Margiotta M (2005) Compendio di psicologia per gli operatori sociosanitari. Piccin, Padova

Imbasciati A, Margiotta M (2008), Psicologia Clinica per gli operatori della salute, Piccin, Padova

Iori V (2006) La nascita: tra scienze umane e scienze mediche. In: La Sala GB, Iori V, Monti F, Fagandini F (a cura di) La "normale" complessità del venire al mondo. Guerini, Milano

Johanson R, Chapman G, Murray D, Jahnson I, Cox J (2000) The North Staffordshire maternity Hospital prospective study of pregnacy-associated depression. Journal of Psychosomatic Obstetric and Gynaecology 21:93-97

Kreppner K (1988) Changes in parent-child relationships with the birth of second child. Marriage \& Family Review 12:157-181

Kreppner K, Paulsen S, Schuetze Y (1982) Infant and Family development: from triads to tetrads. Human Development 25:373-391

Lamb ME (1975) Fathers: forgotten contributors of child development. Human Development 18:245-266

Laplante DP, Barr RG et al (2004) Stress during pregnancy affects general intellectual and language functioning in human toddlers. Pediatr Res 56:400-410 
Lee AM, Lam SK et al (2007) Prevalence, Course, and Risk Factors for Antenatal Anxiety and Depression. Obstetrics \& Gynecology 110:1102-1112

Levey L, Ragan K et al (2004) Psychiatric disorders during pregnancy. Neurology Clinic 22:863893

Lewis JM (1989) The birth of the family: an empirical inquiry. Brunner-Mazel, Philadelphia

Lewis JM, Beavers WR, Gosset JT, Phillips VA (1976) No Single Thread. Psychological Health in Family Systems. Brunner/Mazel, New York

Lindgreen K (2001) Relationship among maternal-fetal attachment, prenatal depression and health practices in pregnancy. Research in Nursing and Health 24:203-217

Lou HC, Hansen D et al (1994) Prenatal stressors of human life affect fetal brain development. Developmental Medicine and Child Neurology 36:826-832

Mastella M (2004) Nascita del figlio e dinamica della coppia: una prospettiva psicoanalitica. In: Arfelli Galli A, Galli G (a cura di) Interpretazione e nascita. La nascita dei genitori-la nascita del figlio. Istituzioni Editoriali e Poligrafici Internazionali, Pisa

Matthey S (2004) Detection and treatment of postnatal depression (perinatal depression or anxiety). Current Opinion in Psychiatry 17:21-29

McHale JP (1995) Co-parenting and triadic interactions during infancy: The roles of marital distress and child gender. Developmental Psychology 31:985-996

McHale JP (2010) La sfida della cogenitorialità. Cortina, Milano

McHale J, Kuersten-Hogan R, Lauretti A (2000) Evaluating coparenting and family-level dynamics during infancy and early childhood: The Coparenting and Family Rating System. In: Kerig P, Lindahl K (a cura di), Family Observational Coding Systems: Resources for Systemic Research. Erlbaum, NJ

McHale JP, Lauretti A, Talbot J, Pouquette C (2002). Retrospect and prospect in the psychological study of coparenting and family group process. In: McHale J, Grolnick W (a cura di) Retrospect and prospect in the psychological study of families. Erlbaum, NJ

McHale JP, Kazali C et al(2004) The transition tocoparenthood: parents'prebirth expectations and early coparental adjustment at three month post-partum. Developmental and Psychopathology 16:711-733

McHale JP, Kuersten-Hogan R, Rao N (2004) Growing points for coparenting theory and research. Journal of adult Development 11:221-234

McHale JP, Rotman T (2007) Expectant parents outlooks on coparenting and later coparenting solidarity. Infant Behavior Development 30:63-81

Mennes M, Stiers P, Lagae L, Van den Bergh B (2006). Long-term cognitive sequelae of antenatal maternal anxiety: involvement of the orbitofrontal cortex. Neurosci Biobehav Rev 30:1078-1086

Milgrom J, Gemmill AW, Bilszta JL, Hayes B, Barnett B, Brooks J, Ericksen J, Ellwood D, Buist A (2008) Antenatal risk factors for postnatal depression: a large prospective study. Journal of Affective Dsorders 108:147-157

Minuchin S (1974) Famiglia e terapia della famiglia. Astrolabio, Roma, 1977

Misri S, Kostaras X, Fox D, Kostaras D (2000) The impact of partner support in the treatment of post-partum depression. Canadian Journal of Psychiatry 45:554-558

Missioner S (2005) La consultazione terapeutica perinatale. Cortina, Milano

Monti F, Agostini F (2006) La Depressione post-partum. Carocci, Roma

Murray-Cooper (1999) Depressione del post-partum e sviluppo del bambino. CIC Edizioni Internazionali, Roma

Nicol-Harper R, Harvey AG, Stein A (2007). Interactions between mothers and infants: impact of maternal anxiety. Infant Behavioral Development 30:161-167

O'Hara MW, Swain AM (1996) Rates and risks of postpartum depression: a meta-analysis. Int Rev Psychiatry 8:37-54

O'Connor TG, Heron J, Golding J et al (2002). Maternal antenatal anxiety and children's behavioral/emotional problems at age 4 years. British Journal of Psychiatry 180:502-508

O'Connor TG, Heron J, Golding J, Glover V, ALSPAC Study Team (2003). Maternal antenatal anxiety and behavioural/emotional problems in children: a test of a programming hypothesis. 
J Child Psychol Psychiatry 44:1025-1036

Pariante C (2012) La depressione post-partum: un problema per entrambi i generi? Seminario "Depressione e psicosi post-partum: fattori di rischio". Facoltà di medicina e chirurgia, Università degli Studi di Brescia, 26 marzo 2012 (in stampa)

Pines D (1972) Pregnancy and motherhood: interaction between fantasy and reality. British Journal of Medical Psychology 45:333-343

Pines D (1977) On becoming a parent. The Israel Annals of Psychiatry and Related Disciplines $15: 2$

Pines D (1982) The revelance of early psychic development to pregnancy and abortion. International Journal of Psychoanalysis 63:311-319

Reck C, Struben K et al (2008) Prevalence, onset and comorbidity of postpartum anxiety and depressive disorders. Acta Psychiatr Scandinavian 118:459-468

Reck C, Zimmer K, Gawlik D (2010) The impact of pre-partum anxiety on the course of childbirth. Poster 12th World Congress World Association for Infant Mental Health (WAIMH) "Infancy in Times of Transition", Leipzig, Germany, 29th June-3rd July

Riva Crugnola C, Baioni E (2002) La comunicazione emotiva del bambino. Età evolutiva, 72:118128

Robertson E, Grace S, Wallington T, Stewart DE (2004) Antenatal risk factors for post-partum Rodriguez A, Bohlin G. Are maternal smoking and stress during pregnancy related to ADHD symptoms in children? Journal of Child Psychology and Psychiatry 46:246-254

Rodriguez A, Bohlin G (2005) Are maternal smoking and stress during pregnancy related to ADHD symptoms in children? Journal of Child Psychology Psychiatry 46:246-254

Ross L, Gilbert Evans S, Sellers E, Romach M (2003). Measurement issues in postpartum depression part 1: anxiety as a feature of postpartum depression. Arch Women Mental Health 6:51-57

Ruiz RJ, Avant KC (2005) Effects of Maternal Prenatal Stress on Infant Outcome. Advances in Nursing Science 28:345-355

Scabini E (1995) Psicologia sociale della famiglia. Boringhieri, Torino

Scabini E, Cigoli V (1991) L'identità organizzativa della famiglia. In: Donati P, Scabini E (a cura di) Identità adulte e relazioni familiari. Studi interdisciplinari sulla famiglia, Vita e Pensiero, Milano

Sifneos P (1973) The prevalence of Alexithymic characteristic in psychosomatic patients. Psychoterapy Psychosomatics 22:255-262

Simonelli A, Fava-Vizziello G, Bighin M, De Palo F, Petech E (2007) La transizione alla triade fra assunzione della genitorialità e riorganizzazione della coppia. Età evolutiva 86:92-99

Stern DN (1985) Il mondo interpersonale del bambino. Boringhieri, Torino, 1987

Stern DN (1995) La costellazione materna. Boringhieri, Torino

Stern DN, Bruschweiler-Stern N (1998) Nascita di una madre. Mondadori, Milano

Stoleru S (1995) Le couple et le projet d'enfant. L'étape initiale du passage à la parentalité. Neuropsichiatrie de l'enfant et de l'adolescent 43:164-170

Talbot J, Elliston D, Thompson J, Scull C, Lieberson R, McHale J (2006) Do early coparenting dynamics benefit from coherent states of mind with respect to attachment? Infant Mental Health Journal 27:43

Taylor GJ, Bagby RM, Parker JDA (1997) I disturbi della regolazione affettiva. Tr. it. Fioriti, Roma, 2000

van Den Bergh BRH, Mulder JH, Mennes M, Glover V (2005) Antenatal maternal anxiety and stress and the neurobehavioural development of the fetus and child: links and possible mechanisms. A review. Neuroscience and Biobehavioral Reviews 29:237-258

Vegetti Finzi S (1990) Il bambino della notte. Mondadori, Milano

Volling BL, Elins JL (1998) Family relationships and children's emotional adjustement as correlates of maternal and paternal differential treatment: a replication with toddler and preschool siblings. Child Development 69:1640-1656

Warren SL, Gunnar MR et al (2003) Maternal panic disorder: infant temperament, neurophysiology, and parenting behaviors. Journal American Academy Child Adolescent Psychology 42:814-825 
3 Werner EA, Myers MM et al (2007) Prenatal predictors of infant temperament. Dev Psychobiol 49:474-484

Winnicott DW (1987) I bambini e le loro madri. Cortina, Milano

Zaslow MJ, Pederesen FA, Suwalsky JT, Rabinovich BA (1986) Fathering during the infancy period. Implications of the mother's employment role. Infant Mental Health Journal 7:225-234

Zaslow MJ, Pederesen FA, Suwalsky JT, Rabinovich BA (1989) Maternal employment and parent-infant interaction at one year. Early Chilhood Research Quarterly 4:459-478

Zeanah PD, Zeananh CH, Stewart L (1990) Parent's Constructions of Their Infant Personalities before and after the Birth: A Descriptive Study. Child Psychiatry and Human Development 20:191-206 